\title{
SOME DECAY ESTIMATES OF SOLUTIONS FOR THE 3-D COMPRESSIBLE ISENTROPIC MAGNETOHYDRODYNAMICS*
}

\author{
JIANWEN ZHANG ${ }^{\dagger}$ AND JUNNING ZHAO ${ }^{\ddagger}$
}

\begin{abstract}
This paper is concerned with the time-asymptotic behavior of solutions to the threedimensional magnetohydrodynamics (MHD) for viscous compressible isentropic fluids. By exploiting some $L^{p}-L^{q}$ estimates of solutions for the heat equation and the linearized Navier-Stokes system, the optimal decay estimates of the solution in $L^{q}$ with $2 \leq q \leq 6$ and its first order derivative in $L^{2}$ are obtained when the initial perturbation around a constant state is sufficiently small in $H^{3}$ and is bounded in $L^{p}$ with any given $1 \leq p<6 / 5$. As a byproduct, the global existence theorem is also proved.
\end{abstract}

Key words. compressible isentropic MHD, decay estimates, $L^{p}-L^{q}$ estimates, global existence.

AMS subject classifications. 76W05, 76N10, 35B40, 35B45.

\section{Introduction}

Magnetohydrodynamics (MHD) concerns the motion of a conducting fluid (plasma) in an electromagnetic field with a very wide range of applications. Due to the interaction between the dynamic motion of the fluid and the evolutions of the magnetic field, the hydrodynamic and electrodynamic effects are strongly coupled. The governing equations for three-dimensional compressible magnetohydrodynamic flows, derived from fluid mechanics with appropriate modifications to account for electrical forces, have the following form (see, e.g., [3, 15, 30, 31, 42]):

$$
\left\{\begin{array}{l}
\rho_{t}+\operatorname{div}(\rho \boldsymbol{u})=0, \\
(\rho \boldsymbol{u})_{t}+\operatorname{div}(\rho \boldsymbol{u} \otimes \boldsymbol{u})+\nabla p(\rho)=(\nabla \times \boldsymbol{B}) \times \boldsymbol{B}+\lambda \Delta \boldsymbol{u}+\left(\lambda+\lambda^{\prime}\right) \nabla \operatorname{div} \boldsymbol{u}, \\
\boldsymbol{B}_{t}-\nabla \times(\boldsymbol{u} \times \boldsymbol{B})=-\nabla \times(\nu \nabla \times \boldsymbol{B}), \quad \operatorname{div} \boldsymbol{B}=0,
\end{array}\right.
$$

where the unknown functions $\rho, \boldsymbol{u} \in \mathbb{R}^{3}, \boldsymbol{B} \in \mathbb{R}^{3}$, and $p=p(\rho)$ are the density, the velocity, the magnetic field, and the pressure of flows, respectively, $\lambda$ and $\lambda^{\prime}$ are the viscosity coefficients of flows satisfying $\lambda>0$ and $2 \lambda / 3+\lambda^{\prime} \geq 0$, and $\nu>0$ is the resistivity constant acting as the magnetic diffusion coefficient of magnetic field.

There have been a lot of studies on MHD by many physicists and mathematicians due to its physical importance, complexity, rich phenomena, and mathematical challenges; see, for example, [3]-[5], [11]-[13], [15], [18]-[20], [23]-[26], [30, 31], [41]-[43], and the references cited therein. In particular, when there is no electromagnetic field, that is, $\boldsymbol{B} \equiv 0$, the system (1.1) reduces to the compressible Navier-Stokes equations for isentropic fluids, which have been studied by many researchers; see, for example, $[14,33,36,38]$ among others. Compared with the Navier-Stokes equations, the presence of magnetic field and its interaction with the hydrodynamic motion in MHD flows

*Received: July 3, 2009; accepted: November 4, 2009. Communicated by Zhouping Xin. The first author was partly supported by the National Natural Science Foundation of China (Grant No. 10801111, 10601008) and the second author was partly supported by the National Natural Science Foundation of China (Grant No. 10971171).

${ }^{\dagger}$ Corresponding author. School of Mathematical Sciences, Xiamen University, Xiamen 361005, China (jwzhang@xmu.edu.cn).

${ }^{\ddagger}$ School of Mathematical Sciences, Xiamen University, Xiamen 361005, China (jnzhao@xmu. edu.cn). 
will cause serious difficulties. Thus, the extension of results known for the compressible Navier-Stokes equations to the magnetohydrodynamic equations doesn't always appear a simple matter. For example, even for the one-dimensional case, the global smooth (classical) solution to the full perfect MHD equations with large initial data is still unknown when all the viscosity, heat conductivity, and magnetic diffusivity coefficients are constants, although the corresponding problem of the Navier-Stokes equations for compressible fluids was successfully solved by Kazhikhov and Shelukhin (cf. [27]) about thirty years ago.

In this paper, we consider an initial value problem of (1.1) in the whole space $\mathbb{R}^{3}$ with the initial data

$$
(\rho, \boldsymbol{u}, \boldsymbol{B})(\boldsymbol{x}, 0)=\left(\rho_{0}, \boldsymbol{u}_{0}, \boldsymbol{B}_{0}\right)(\boldsymbol{x}) \rightarrow\left(\rho_{\infty}, 0,0\right) \quad \text { as } \quad|\boldsymbol{x}| \rightarrow \infty,
$$

where $\rho_{\infty}>0$ is a given constant. It is assumed throughout this paper that $p=p(\rho)$ is smooth in a neighborhood of $\rho_{\infty}$ with $p^{\prime}\left(\rho_{\infty}\right)>0$.

The main purpose of this paper is to study the decay estimates of solutions to the initial value problem (1.1) and (1.2). For this purpose, we need the following proposition concerning the global existence of solutions.

Proposition 1.1. (Global existence) There exists a constant $\varepsilon>0$ such that if the initial data satisfies

$$
\left\|\left(\rho_{0}-\rho_{\infty}, \boldsymbol{u}_{0}, \boldsymbol{B}_{0}\right)\right\|_{3} \leq \varepsilon
$$

then the initial value problem (1.1) and (1.2) admits a unique global-in-time solution $(\rho, \boldsymbol{u}, \boldsymbol{B})$ satisfying $\rho>0$ and

$$
\begin{gathered}
\rho-\rho_{\infty} \in C\left(0, \infty ; H^{3}\left(\mathbb{R}^{3}\right)\right) \cap C^{1}\left(0, \infty ; H^{2}\left(\mathbb{R}^{3}\right)\right), \\
\boldsymbol{u}, \boldsymbol{B} \in C\left(0, \infty ; H^{3}\left(\mathbb{R}^{3}\right)\right) \cap C^{1}\left(0, \infty ; H^{1}\left(\mathbb{R}^{3}\right)\right) .
\end{gathered}
$$

Moreover, there exists a constant $K>0$ such that for any $t \geq 0$, it holds that

$$
\left\|\left(\rho-\rho_{\infty}, \boldsymbol{u}, \boldsymbol{B}\right)(t)\right\|_{3}^{2}+\int_{0}^{t}\left(\|\nabla \rho(\tau)\|_{2}^{2}+\|\nabla(\boldsymbol{u}, \boldsymbol{B})(\tau)\|_{3}^{2}\right) d \tau \leq K\left\|\left(\rho_{0}-\rho_{\infty}, \boldsymbol{u}_{0}, \boldsymbol{B}_{0}\right)\right\|_{3}^{2} .
$$

In [23], Kawashima considered the global smooth solutions of the two-dimensional MHD when the initial data is close to a constant state. So, Proposition 1.1 extends Kawashima's existence result to the three-dimensional magnetohydrodynamic equations for viscous compressible isentropic fluids.

Our main aim is to prove some decay properties of the solution obtained in Proposition 1.1 under the additional condition that the initial perturbation $\left(\rho_{0}-\rho_{\infty}, \boldsymbol{u}_{0}, \boldsymbol{B}_{0}\right)$ is bounded in $L^{p}$ with any given $p \in[1,6 / 5)$. More precisely, our main result can be stated as follows.

Theorem 1.2. (Decay estimates) Let the conditions of Proposition 1.1 be satisfied and let $(\rho, \boldsymbol{u}, \boldsymbol{B})$ be a global classical solution in $H^{3}\left(\mathbb{R}^{3}\right)$ of the problem (1.1), (1.2). Assume further that

$$
\left\|\left(\rho_{0}-\rho_{\infty}, \boldsymbol{u}_{0}, \boldsymbol{B}_{0}\right)\right\|_{L^{p}}<\infty, \quad 1 \leq p<6 / 5 .
$$

Then there exist constants $\varepsilon_{0}>0$ and $K_{0}>0$ such that for any $0<\varepsilon \leq \varepsilon_{0}$, it holds that

$$
\begin{array}{r}
\left\|\nabla\left(\rho-\rho_{\infty}, \boldsymbol{u}, \boldsymbol{B}\right)(t)\right\|_{2} \leq K_{0}(1+t)^{-\sigma(p, 2 ; 1)}, \\
\left\|\partial_{t}(\rho, \boldsymbol{u}, \boldsymbol{B})(t)\right\|_{1} \leq K_{0}(1+t)^{-\sigma(p, 2 ; 1)},
\end{array}
$$


and

$$
\left\|\left(\rho-\rho_{\infty}, \boldsymbol{u}, \boldsymbol{B}\right)(t)\right\|_{L^{q}} \leq K_{0}(1+t)^{-\sigma(p, q ; 0)}, \quad 2 \leq q \leq 6,
$$

where the decay exponent $\sigma(p, q ; k)$ is given by

$$
\sigma(p, q ; k)=\frac{3}{2}\left(\frac{1}{p}-\frac{1}{q}\right)+\frac{k}{2} .
$$

A lot of works on the time-asymptotic behavior of solutions for the compressible Navier-Stokes equations have been done for either isentropic or heat-conductive fluids, see [6]-[9], [16, 17, 22, 21, 28, 29, 34, 40] and the references cited therein. Here, we would like to mention the papers $[8,9,40]$, in which the authors considered the convergence rates of solutions to the compressible Navier-Stokes equations with potential external force. Roughly speaking, under the analogous conditions in Theorem 1.2 on initial data, as well as the smallness conditions on the external force in some Sobolev spaces, the (almost) optimal convergence rates of solutions for compressible Navier-Stokes equations were obtained in $[8,9,40]$ by using the energy estimates and some $L^{p}-L^{q}$ estimates (cf. [28, 29]) for the linearized Navier-Stokes system. However, compared with these works, the situation for the MHD problem is completely different due to the additional presence of magnetic field and its strong interaction on the hydrodynamic motion. The reason is that the magnetic field $\boldsymbol{B}$ is an unknown function depending strongly on the velocity $\boldsymbol{u}$, so we can not treat the additional nonlinear term $(\nabla \times \boldsymbol{B}) \times \boldsymbol{B}$ (acting as the magnetic pressure) in (1.1) 2 as the (known) potential external force in the Navier-Stokes equations. To overcome this difficulty, we observe that the equation $(1.1)_{3}$ for the magnetic field,

$$
\boldsymbol{B}_{t}-\nu \Delta \boldsymbol{B}=\nabla \times(\boldsymbol{u} \times \boldsymbol{B})
$$

is in the form of linear parabolic equation with a nonhomogeneous term $\nabla \times(\boldsymbol{u} \times \boldsymbol{B})$. Thus, by using Young's inequality and the estimates of the standard heat kernel, we can obtain some a priori decay properties of $\boldsymbol{B}$ from the above equation. These decay estimates strongly depend on the derivatives $\nabla \boldsymbol{u}$ and $\nabla \boldsymbol{B}$. Combining these estimates with the $L^{p}-L^{q}$ estimates of solutions from spectral analysis on the linearized NavierStokes system (cf. $[28,29]$ ), we can prove the decay estimates stated in Theorem 1.2 in a manner similar to that in $[8,9,40]$.

REMARK 1.3. By comparison, we find that the decay rates for the solution in $L^{q}$-norm with $2 \leq q \leq 6$ and the one for the first order derivative in $L^{2}$-norm are the same as those for the linearized Navier-Stokes system (see Lemma 2.3) and the heat equation (see [39]). Therefore, these decay estimates are optimal.

REMARK 1.4. It is worth pointing out that the constant $K_{0}$ in Theorem 1.2 depends only on $p$ and $\left\|\left(\rho_{0}-\rho_{\infty}, \boldsymbol{u}_{0}, \boldsymbol{B}_{0}\right)\right\|_{L^{p} \cap H^{3}}$. Moreover, we also note that $p=6 / 5$, which comes from the inequality $\sigma(p, 2 ; 1)>1$ when $p<6 / 5$, is the critical case. In other words, the constant $K_{0}$ may tend to infinity when $p$ approaches $6 / 5$.

In the remainder of this section, we reformulate our problem. To do so, we introduce the change of unknown functions

$$
(\rho, \boldsymbol{u}, \boldsymbol{B}) \rightarrow\left(\rho+\rho_{\infty}, \alpha \boldsymbol{u}, \boldsymbol{B}\right) \quad \text { with } \quad \alpha=\sqrt{p^{\prime}\left(\rho_{\infty}\right)} / \rho_{\infty} .
$$

Here and hereafter, for the notational simplicity we still denote the transformed functions by $\rho, \boldsymbol{u}$, and $\boldsymbol{B}$, and their corresponding initial data by $\rho_{0}, \boldsymbol{u}_{0}$, and $\boldsymbol{B}_{0}$. Then, 
the initial value problem (1.1)-(1.2) in $\mathbb{R}^{3} \times \mathbb{R}_{+}$is reduced to the following problem:

$$
\left\{\begin{array}{l}
\rho_{t}+\beta \operatorname{div} \boldsymbol{u}=f, \\
\boldsymbol{u}_{t}-\tilde{\lambda} \Delta \boldsymbol{u}-\tilde{\mu} \nabla \operatorname{div} \boldsymbol{u}+\beta \nabla \rho=\boldsymbol{g}, \\
\boldsymbol{B}_{t}-\nu \Delta \boldsymbol{B}=\boldsymbol{h}, \quad \operatorname{div} \boldsymbol{B}=0,
\end{array}\right.
$$

with initial conditions

$$
(\rho, \boldsymbol{u}, \boldsymbol{B})(\boldsymbol{x}, 0)=\left(\rho_{0}, \boldsymbol{u}_{0}, \boldsymbol{B}_{0}\right)(\boldsymbol{x}) \rightarrow(0,0,0) \quad \text { as } \quad|\boldsymbol{x}| \rightarrow \infty,
$$

where $\tilde{\lambda}=\lambda / \rho_{\infty}, \tilde{\mu}=\mu / \rho_{\infty}, \mu=\lambda+\lambda^{\prime}, \beta=\sqrt{p^{\prime}\left(\rho_{\infty}\right)}$ are positive constants, and

$$
\begin{aligned}
f= & -\alpha \rho \operatorname{div} \boldsymbol{u}-\alpha(\boldsymbol{u} \cdot \nabla) \rho, \\
\boldsymbol{g}= & \left(\frac{\lambda}{\rho+\rho_{\infty}}-\frac{\lambda}{\rho_{\infty}}\right) \Delta \boldsymbol{u}+\left(\frac{\mu}{\rho+\rho_{\infty}}-\frac{\mu}{\rho_{\infty}}\right) \nabla \operatorname{div} \boldsymbol{u}-\alpha(\boldsymbol{u} \cdot \nabla) \boldsymbol{u} \\
& -\frac{1}{\alpha}\left(\frac{p^{\prime}\left(\rho+\rho_{\infty}\right)}{\rho+\rho_{\infty}}-\frac{p^{\prime}\left(\rho_{\infty}\right)}{\rho_{\infty}}\right) \nabla \rho+\frac{\alpha^{-1}}{\rho+\rho_{\infty}}(\nabla \times \boldsymbol{B}) \times \boldsymbol{B}, \\
\boldsymbol{h}= & \alpha(\boldsymbol{B} \cdot \nabla) \boldsymbol{u}-\alpha(\boldsymbol{u} \cdot \nabla) \boldsymbol{B}-\alpha \boldsymbol{B} \operatorname{div} \boldsymbol{u} .
\end{aligned}
$$

Clearly, for smooth solutions in $H^{3}\left(\mathbb{R}^{3}\right)$, the initial value problem of $(1.1),(1.2)$ are equivalent to that of $(1.4),(1.5)$. Thus, to prove Theorem 1.2, it suffices to show the following proposition.

Proposition 1.5. Let $(\rho, \boldsymbol{u}, \boldsymbol{B})$ be a global smooth solution in $H^{3}\left(\mathbb{R}^{3}\right)$ to the initial value problem (1.4) and (1.5). Assume that for some small constant $\varepsilon>0$, there holds

$$
\left\|\left(\rho_{0}, \boldsymbol{u}_{0}, \boldsymbol{B}_{0}\right)\right\|_{3} \leq \varepsilon
$$

and that for any given $1 \leq p<6 / 5$, there holds

$$
\left\|\left(\rho_{0}, \boldsymbol{u}_{0}, \boldsymbol{B}_{0}\right)\right\|_{L^{p}}<\infty .
$$

Then there are positive constants $\varepsilon_{0}$ and $K_{0}$ such that for any $0 \leq \varepsilon \leq \varepsilon_{0}$, we have

$$
\begin{gathered}
\|\nabla(\rho, \boldsymbol{u}, \boldsymbol{B})(t)\|_{2} \leq K_{0}(1+t)^{-\sigma(p, 2 ; 1)} \\
\left\|\partial_{t}(\rho, \boldsymbol{u}, \boldsymbol{B})(t)\right\|_{1} \leq K_{0}(1+t)^{-\sigma(p, 2 ; 1)}
\end{gathered}
$$

and

$$
\|(\rho, \boldsymbol{u}, \boldsymbol{B})(t)\|_{L^{q}} \leq K_{0}(1+t)^{-\sigma(p, q ; 0)}, \quad 2 \leq q \leq 6,
$$

where $\sigma(p, q ; k)$ is the same as in (1.3).

The paper is organized as follows. Some a priori decay estimates on the magnetic field and some elementary inequalities are proved in section 2, where the well-known Sobolev inequalities and Moser-type calculus inequalities are also recalled. The main results are proved in section 3, where we first derive some global a priori estimates in subsection 3.1, then prove the global existence (Proposition 1.1) in subsection 3.2, and finally give the proof of Proposition 1.5 and complete the proof of Theorem 1.2 in subsection 3.3 . 
Notation 1.6. Let $H^{k}\left(\mathbb{R}^{3}\right)$ and $W^{k, p}\left(\mathbb{R}^{3}\right)$ with $k \in \mathbb{Z}^{+}, p \geq 1$ be the usual Sobolev spaces with norms denoted by $\|\cdot\|_{k}$ and $\|\cdot\|_{k, p}$, respectively. In particular, when $k=0$, we denote the norms by $\|\cdot\|_{L^{2}}$ and $\|\cdot\|_{L^{p}}$, respectively. $\langle\cdot, \cdot\rangle$ is used to denote the $L^{2}$ inner product. As usual, $\nabla=\left(\partial_{1}, \partial_{2}, \partial_{3}\right), \partial_{i}=\partial_{x_{i}}, i=1,2,3$, and $\nabla^{l} f$ denotes all the derivatives of $l$-th order on $f$. For a multi-index $k=\left(k_{1}, k_{2}, k_{3}\right)$ with $|k|=k_{1}+k_{2}+k_{3}$, we set $D^{k}=\partial_{1}^{k_{1}} \partial_{2}^{k_{2}} \partial_{3}^{k_{3}}$. For simplicity, we shall use $C$ and $C_{i}(i=1,2,3, \ldots)$ to denote various positive constants which may vary from line to line.

\section{Some auxiliary lemmas}

In this section, we list some auxiliary lemmas which will be used later. First of all, we recall the Young inequality which can be found in [2].

Lemma 2.1. (Young inequality). For $1 \leq r \leq \infty, 1 \leq p \leq r^{\prime}$, and $r^{\prime}=r /(r-1)$, assume that $K \in L^{r}\left(\mathbb{R}^{n}\right)$ and $f \in L^{p}\left(\mathbb{R}^{n}\right)$. Then,

$$
\|K * f\|_{L^{q}} \leq\|K\|_{L^{r}}\|f\|_{L^{p}},
$$

where “*” denotes convolution, and $q>0$ satisfies

$$
\frac{1}{q}=\frac{1}{p}+\frac{1}{r}-1 .
$$

With the help of Lemma 2.1, we can make use of the standard heat kernel to deduce some a priori decay properties of the magnetic field from equation (1.4) $)_{3}$.

LEMma 2.2. For smooth functions $\boldsymbol{B}_{0}, \boldsymbol{h}(\cdot, t) \in L^{p}\left(\mathbb{R}^{3}\right) \cap H^{3}\left(\mathbb{R}^{3}\right)$ with $1 \leq p \leq 2$, let $\boldsymbol{B}=\boldsymbol{B}(\boldsymbol{x}, t)$ be a smooth solution of $(1.4)_{3}$ with initial data $\boldsymbol{B}_{0}$. Then for $k=1,2,3$, it holds that

$$
\begin{aligned}
\left\|\nabla^{k} \boldsymbol{B}(t)\right\|_{L^{2}} \leq & C(1+t)^{-\sigma(p, 2 ; k)}\left\|\boldsymbol{B}_{0}\right\|_{L^{p} \cap H^{k}} \\
& +C \int_{0}^{t}(1+t-\tau)^{-\sigma(p, 2 ; k)}\|\boldsymbol{h}(\tau)\|_{L^{p} \cap H^{k}} d \tau, \quad \forall t \geq 0,
\end{aligned}
$$

where $C>0$ depends only on $p$ and $k$ and $\sigma(p, 2 ; k)$ is defined as in (1.3).

Proof. By scaling technique, we may assume that $\nu \equiv 1$ in (1.4) $)_{3}$. It follows from $(1.4)_{3}$ and the classical theory of linear parabolic equations (see, for example, [39]) that the magnetic field $\boldsymbol{B}$ is given by

$$
\boldsymbol{B}(\boldsymbol{x}, t)=S(t) \boldsymbol{B}(0)+\int_{0}^{t} S(t-\tau) \boldsymbol{h}(\cdot, \tau) d \tau,
$$

where $S(t): \varphi \rightarrow u(\cdot, t)$ is the solution semigroup defined by $S(t)=e^{-t \Delta}$, that is,

$$
u(\boldsymbol{x}, t)=S(t) \varphi=\frac{1}{(2 \sqrt{\pi t})^{3}} \int_{\mathbb{R}^{3}} \exp \left\{-\frac{\left|\boldsymbol{x}-\boldsymbol{x}^{\prime}\right|^{2}}{4 t}\right\} \varphi\left(\boldsymbol{x}^{\prime}\right) d \boldsymbol{x}^{\prime} .
$$

Clearly, $u(x, t)=S(t) \varphi$ is the unique solution of the heat equation

$$
\left\{\begin{array}{lc}
u_{t}-\Delta u=0, & (\boldsymbol{x}, t) \in \mathbb{R}^{3} \times \mathbb{R}_{+}, \\
u(\boldsymbol{x}, 0)=\varphi(\boldsymbol{x}), & \boldsymbol{x} \in \mathbb{R}^{3} .
\end{array}\right.
$$

Moreover,

$$
\nabla^{k} u=\nabla^{k}(S(t) \varphi)=\nabla^{k} K(t) * \varphi,
$$


where $K:=K(t)$ is the heat kernel

$$
K(\boldsymbol{x}, t)=\frac{1}{(2 \sqrt{\pi t})^{3}} \exp \left\{-\frac{|\boldsymbol{x}|^{2}}{4 t}\right\} .
$$

Thanks to the estimate $\|K(t)\|_{L^{1}}=1$, we have from a direct computation that

$$
\left\|\nabla^{k} K(t)\right\|_{L^{r}} \leq C t^{-(3 / 2)(1-1 / r)-k / 2}, \quad k=1,2,3 .
$$

From this and the Young inequality in Lemma 2.1 with $q=2$ and $1 / r=1+1 / 2-1 / p$, we obtain that

$$
\begin{aligned}
\left\|\nabla^{k} u(t)\right\|_{L^{2}} & =\left\|\nabla^{k} K(t) * \varphi\right\|_{L^{2}} \leq\left\|\nabla^{k} K(t)\right\|_{L^{r}}\|\varphi\|_{L^{p}} \\
& \leq C t^{-(3 / 2)(1-1 / r)-k / 2}\|\varphi\|_{L^{p}}=C t^{-\sigma(p, 2 ; k)}\|\varphi\|_{L^{p}} .
\end{aligned}
$$

On the other hand, using the standard energy method, we find

$$
\left\|\nabla^{k} u(t)\right\|_{L^{2}}=\left\|\nabla^{k} S(t) \varphi\right\|_{L^{2}} \leq\left\|\nabla^{k} \varphi\right\|_{L^{2}}, \quad k=1,2,3 .
$$

Collecting these two estimates together, we conclude that

$$
\left\|\nabla^{k} S(t) \varphi\right\|_{L^{2}} \leq C(1+t)^{-\sigma(p, 2 ; k)}\left(\|\varphi\|_{L^{p}}+\|\varphi\|_{k}\right), \quad k=1,2,3 .
$$

Applying the above estimates to the integral formula (2.1), we immediately obtain the desired estimates of Lemma 2.1.

To deal with $\rho$ and $\boldsymbol{u}$, we recall the following lemma concerning the decay properties of the solution to the linearized system of $(1.4)_{1}$ and $(1.4)_{2}$ (see $[17,28,29]$ ).

LEMmA 2.3. Let $k \geq 0$ be an integer and $1 \leq p \leq 2 \leq q<\infty$. Then for any $t \geq 0$, it holds that

$$
\left\|\nabla^{k} E(t) \boldsymbol{U}(0)\right\|_{L^{q}} \leq C(1+t)^{-\sigma(p, q ; k)}\|\boldsymbol{U}(0)\|_{L^{p} \cap H^{k}},
$$

where $\sigma(p, q ; k)$ is the same as in (1.3), $\boldsymbol{U}(t):=E(t) \boldsymbol{U}(0)$ is the solution of the linear equations

$$
\boldsymbol{U}_{t}+\mathbb{A} \boldsymbol{U}=0
$$

with

$$
\mathbb{A}=\left(\begin{array}{lc}
0 & \beta d i v \\
\beta \nabla & -\tilde{\lambda} \Delta-\tilde{\mu} \nabla d i v
\end{array}\right),
$$

and $E(t)=e^{-t \mathbb{A}}$ is the solution semigroup generated by $-\mathbb{A}$.

By virtue of Lemmas 2.3 and Duhamel's principle, we have

LemMA 2.4. For any given $1 \leq p \leq 2$, assume that $\rho_{0}, \boldsymbol{u}_{0}, f, \boldsymbol{g} \in L^{p}\left(\mathbb{R}^{3}\right) \cap H^{3}\left(\mathbb{R}^{3}\right)$. Let $(\rho, \boldsymbol{u})$ be a smooth solution of equations (1.4) $)_{1}$ and $(1.4)_{2}$ with initial data $\left(\rho_{0}, \boldsymbol{u}_{0}\right)$. Then for $k=1,2,3$, it holds that

$$
\begin{aligned}
\left\|\nabla^{k}(\rho, \boldsymbol{u})(t)\right\|_{L^{2}} \leq & C(1+t)^{-\sigma(p, 2 ; k)}\left\|\left(\rho_{0}, \boldsymbol{u}_{0}\right)\right\|_{L^{p} \cap H^{k}} \\
& +C \int_{0}^{t}(1+t-\tau)^{-\sigma(p, 2 ; k)}\|(f, \boldsymbol{g})(\tau)\|_{L^{p} \cap H^{k}} d \tau, \quad \forall t \geq 0,
\end{aligned}
$$


where $C>0$ depends only on $p$ and $k$, and $\sigma(p, 2 ; k)$ is given by (1.3) with $q=2$.

Proof. Duhamel's principle implies that the solution $(\rho, \boldsymbol{u})$ of $(1.4)_{1}$ and $(1.4)_{2}$ is given by

$$
(\rho, \boldsymbol{u})(\boldsymbol{x}, t)=E(t)\left(\rho_{0}, \boldsymbol{u}_{0}\right)+\int_{0}^{t} E(t-\tau)(f, \boldsymbol{g})(\tau) \mathrm{d} \tau .
$$

Thus, the application of Lemma 2.3 with $q=2$ immediately leads to Lemma 2.4.

For the derivation of a priori estimates, we need some Sobolev inequalities and the Moser-type calculus inequalities, which are well-known and can be found in [1] and [35], respectively.

LEMMA 2.5 .

(A) (Sobolev inequalities.) For any $f \in H^{2}$ and $q \in[2,6]$, it holds that

$$
\|f\|_{L^{q}} \leq C\|f\|_{1}, \quad\|f\|_{L^{6}} \leq C\|\nabla f\|_{L^{2}}, \quad\|f\|_{L^{\infty}} \leq C\|f\|_{W^{1,6}} \leq C\|\nabla f\|_{1} .
$$

(B) (Commutator estimate.) For $f \in H^{s}, D f \in L^{\infty}$, and $g \in H^{s-1} \cap L^{\infty}$, it holds that

$$
\sum_{|\alpha| \leq s}\left\|D^{\alpha}(f g)-f D^{\alpha} g\right\|_{L^{2}} \leq C\left(\|f\|_{s}\|g\|_{L^{\infty}}+\|D f\|_{L^{\infty}}\|g\|_{s-1}\right) .
$$

(C) (Product estimate.) For $f, g \in H^{s} \cap L^{\infty}$, it holds that

$$
\sum_{|\alpha| \leq s}\left\|D^{\alpha}(f g)\right\|_{L^{2}} \leq C\left(\|f\|_{L^{\infty}}\|g\|_{s}+\|f\|_{s}\|g\|_{L^{\infty}}\right) .
$$

Here, $s \in \mathbb{Z}^{+}$is an integer, and $\alpha$ is the standard multi-index with $|\alpha|=\alpha_{1}+\alpha_{2}+\alpha_{3}$.

Finally, we state the following elementary inequalities, which will be used in the proof of decay estimates.

Lemma 2.6. Let $a, b, c \in \mathbb{R}$ be such that $0 \leq a \leq b, b>1$, and $c>0$. Then for any $t \geq 0$, we have

$$
\begin{gathered}
\int_{0}^{t}(1+t-\tau)^{-a}(1+\tau)^{-b} d \tau \leq C(1+t)^{-a}, \\
\int_{0}^{t}(1+\tau)^{-a} \exp \{-c(t-\tau)\} d \tau \leq C(1+t)^{-a},
\end{gathered}
$$

where $C>0$ only depends on $a, b$, and $c$.

Proof. The proof of the first inequality can be found in [32]. Indeed, a direct computation gives

$$
\begin{aligned}
\int_{0}^{t}(1+t-\tau)^{-a}(1+\tau)^{-b} d \tau= & \left(\int_{0}^{t / 2}+\int_{t / 2}^{t}\right)(1+t-\tau)^{-a}(1+\tau)^{-b} d \tau \\
\leq & \left(1+\frac{t}{2}\right)^{-a} \int_{0}^{t / 2}(1+\tau)^{-b} d \tau \\
& +\left(1+\frac{t}{2}\right)^{-b} \int_{t / 2}^{t}(1+t-\tau)^{-a} d \tau \\
\leq & C(1+t)^{-a}+C(1+t)^{-b} \int_{t / 2}^{t}(1+t-\tau)^{-a} d \tau \\
:= & C(1+t)^{-a}+I,
\end{aligned}
$$


where the second term $I$ can be bounded by

$$
I \leq \begin{cases}C(1+t)^{-b}\left(1-\left(1+\frac{t}{2}\right)^{1-a}\right) \leq C(1+t)^{-b} \leq C(1+t)^{-a}, & \text { if } \quad a>1, \\ C(1+t)^{-b}\left(\left(1+\frac{t}{2}\right)^{1-a}-1\right) \leq C(1+t)^{1-a-b} \leq C(1+t)^{-a}, & \text { if } \quad a<1, \\ C(1+t)^{-b} \ln \left(1+\frac{t}{2}\right) \leq C(1+t)^{-b} \ln (1+t) \leq C(1+t)^{-a}, & \text { if } \quad a=1 .\end{cases}
$$

Here, we have used $b \geq a, b \geq 1$, and the fact that $b>a$ when $a=1$. This completes the proof of the first inequality. As a result, one obtains the second one since it is easy to verify that $(1+t-\tau)^{b} e^{-c(t-\tau)} \leq C$ for any $b \geq 0, c>0$, and $0 \leq \tau \leq t$.

\section{Global existence and decay estimates}

3.1. A priori estimates. Suppose that $(\rho, \boldsymbol{u}, \boldsymbol{B})$ is a solution in $H^{3}\left(\mathbb{R}^{3}\right)$ to the initial value problem (1.4) and (1.5) on the interval $[0, T]$ with some $T>0$. Furthermore, for some $\delta>0$ small enough, we also make the following a priori assumption

$$
\sup _{t \in[0, T]}\|(\rho, \boldsymbol{u}, \boldsymbol{B})(t)\|_{3} \leq \delta .
$$

Then, by virtue of Lemma 2.5 and (3.1), we can prove

Lemma 3.1. For enough small $\delta>0$, there are suitably large constants $K_{1}, K_{2}>0$ such that for any $t \geq 0$ it holds that

$$
\begin{aligned}
& \frac{d}{d t}\left(K_{1}\|(\rho, \boldsymbol{u}, \boldsymbol{B})(t)\|_{L^{2}}^{2}+\langle\nabla \rho, \boldsymbol{u}\rangle\right)+C\|\nabla(\rho, \boldsymbol{u}, \boldsymbol{B})(t)\|_{L^{2}}^{2} \leq C\left\|\nabla^{2} \boldsymbol{u}(t)\right\|_{L^{2}}^{2} \\
& \frac{d}{d t}\left(K_{2}\|\nabla(\rho, \boldsymbol{u}, \boldsymbol{B})(t)\|_{2}^{2}+\sum_{1 \leq|l| \leq 2}\left\langle\nabla D^{l} \rho, D^{l} \boldsymbol{u}\right\rangle\right) \\
& \quad+C\left(\left\|\nabla^{2} \rho(t)\right\|_{1}^{2}+\left\|\nabla^{2}(\boldsymbol{u}, \boldsymbol{B})(t)\right\|_{2}^{2}\right) \leq C \delta\|\nabla(\rho, \boldsymbol{u}, \boldsymbol{B})(t)\|_{L^{2}}^{2} .
\end{aligned}
$$

Proof. Multiplying $(1.4)_{1},(1.4)_{2}$, and $(1.4)_{3}$ by $\rho, \boldsymbol{u}$, and $\boldsymbol{B}$ in $L^{2}$, respectively, and integrating by parts, we obtain after adding them together that

$$
\begin{aligned}
& \frac{1}{2} \frac{d}{d t}\|(\rho, \boldsymbol{u}, \boldsymbol{B})(t)\|_{L^{2}}^{2}+\tilde{\lambda}\|\nabla \boldsymbol{u}\|_{L^{2}}^{2}+\tilde{\mu}\|\operatorname{div} \boldsymbol{u}\|_{L^{2}}^{2}+\nu\|\nabla \boldsymbol{B}\|_{L^{2}}^{2} \\
= & \langle f, \rho\rangle+\langle\boldsymbol{g}, \boldsymbol{u}\rangle+\langle\boldsymbol{h}, \boldsymbol{B}\rangle .
\end{aligned}
$$

The terms on the right-hand side can be bounded as follows, using Sobolev inequalities in Lemma 2.5 (i) and the a priori Assumption (3.1).

$$
\begin{aligned}
&|\langle f, \rho\rangle|=\frac{\alpha}{2}\left|\left\langle\rho^{2}, \operatorname{div} \boldsymbol{u}\right\rangle\right| \leq C\|\rho\|_{L^{3}}\|\rho\|_{L^{6}}\|\operatorname{div} \boldsymbol{u}\|_{L^{2}} \leq C \delta\left(\|\nabla \rho\|_{L^{2}}^{2}+\|\nabla \boldsymbol{u}\|_{L^{2}}^{2}\right), \\
&|\langle\boldsymbol{g}, \boldsymbol{u}\rangle| \leq C\{|\langle\rho \nabla \boldsymbol{u}, \nabla \boldsymbol{u}\rangle|+|\langle\nabla \rho \nabla \boldsymbol{u}, \boldsymbol{u}\rangle|+|\langle\rho \operatorname{div} \boldsymbol{u}, \operatorname{div} \boldsymbol{u}\rangle|+|\langle\nabla \rho \operatorname{div} \boldsymbol{u}, \boldsymbol{u}\rangle| \\
&+|\langle(\boldsymbol{u} \cdot \nabla) \boldsymbol{u}, \boldsymbol{u}\rangle|+|\langle\rho \nabla \rho, \boldsymbol{u}\rangle|+|\langle(\nabla \times \boldsymbol{B}) \times \boldsymbol{B}, \boldsymbol{u}\rangle|\} \\
& \leq C\left\{\|\rho\|_{L^{\infty}}\|\nabla \boldsymbol{u}\|_{L^{2}}^{2}+\|\nabla \rho\|_{L^{3}}\|\nabla \boldsymbol{u}\|_{L^{2}}\|\boldsymbol{u}\|_{L^{6}}+\|\boldsymbol{u}\|_{L^{3}}\|\nabla \boldsymbol{u}\|_{L^{2}}\|\boldsymbol{u}\|_{L^{6}}\right. \\
&\left.+\|\rho\|_{L^{3}}\|\nabla \rho\|_{L^{2}}\|\boldsymbol{u}\|_{L^{6}}+\|\boldsymbol{B}\|_{L^{3}}\|\nabla \boldsymbol{B}\|_{L^{2}}\|\boldsymbol{u}\|_{L^{6}}\right\} \\
& \leq C \delta\left(\|\nabla \rho\|_{L^{2}}^{2}+\|\nabla \boldsymbol{u}\|_{L^{2}}^{2}+\|\nabla \boldsymbol{B}\|_{L^{2}}^{2}\right),
\end{aligned}
$$


and

$$
|\langle\boldsymbol{h}, \boldsymbol{B}\rangle| \leq C\left(\|\nabla \boldsymbol{u}\|_{L^{2}}\|\boldsymbol{B}\|_{L^{6}}+\|\boldsymbol{u}\|_{L^{6}}\|\nabla \boldsymbol{B}\|_{L^{2}}\right)\|\boldsymbol{B}\|_{L^{3}} \leq C \delta\left(\|\nabla \boldsymbol{B}\|_{L^{2}}^{2}+\|\nabla \boldsymbol{u}\|_{L^{2}}^{2}\right) .
$$

Since $\delta>0$ is small enough, plugging these estimates into (3.4), we obtain

$$
\frac{d}{d t}\|(\rho, \boldsymbol{u}, \boldsymbol{B})(t)\|_{L^{2}}^{2}+\|\nabla(\boldsymbol{u}, \boldsymbol{B})(t)\|_{L^{2}}^{2} \leq C \delta\|\nabla \rho(t)\|_{L^{2}}^{2} .
$$

To estimate $\|\nabla \rho\|_{L^{2}}^{2}$, we first observe from $(1.4)_{2}$ that

$$
\beta\|\nabla \rho\|_{L^{2}}^{2}=-\left\langle\boldsymbol{u}_{t}, \nabla \rho\right\rangle+\tilde{\lambda}\langle\Delta \boldsymbol{u}, \nabla \rho\rangle+\tilde{\mu}\langle\nabla \operatorname{div} \boldsymbol{u}, \nabla \rho\rangle+\langle\boldsymbol{g}, \nabla \rho\rangle,
$$

which together with the identity

$$
\left\langle\boldsymbol{u}_{t}, \nabla \rho\right\rangle=\frac{d}{d t}\langle\boldsymbol{u}, \nabla \rho\rangle-\left\langle\boldsymbol{u}, \nabla \rho_{t}\right\rangle=\frac{d}{d t}\langle\boldsymbol{u}, \nabla \rho\rangle+\langle f, \operatorname{div} \boldsymbol{u}\rangle-\beta\|\operatorname{div} \boldsymbol{u}\|_{L^{2}}^{2},
$$

implies that

$$
\beta\|\nabla \rho\|_{L^{2}}^{2}+\frac{d}{d t}\langle\boldsymbol{u}, \nabla \rho\rangle=\langle\tilde{\lambda} \Delta \boldsymbol{u}+\tilde{\mu} \nabla \operatorname{div} \boldsymbol{u}, \nabla \rho\rangle+\langle\boldsymbol{g}, \nabla \rho\rangle-\langle f, \operatorname{div} \boldsymbol{u}\rangle+\beta\|\operatorname{div} \boldsymbol{u}\|_{L^{2}}^{2} .
$$

By virtue of Hölder inequality, Sobolev inequalities, and (3.1), we see that

$$
|\langle\tilde{\lambda} \Delta \boldsymbol{u}+\tilde{\mu} \nabla \operatorname{div} \boldsymbol{u}, \nabla \rho\rangle| \leq \frac{\beta}{2}\|\nabla \rho\|_{L^{2}}^{2}+C\left\|\nabla^{2} \boldsymbol{u}\right\|_{L^{2}}^{2},
$$

and

$$
\begin{aligned}
|\langle\boldsymbol{g}, \nabla \rho\rangle|+|\langle f, \operatorname{div} \boldsymbol{u}\rangle| \leq & C\|(\rho, \boldsymbol{u}, \boldsymbol{B})\|_{L^{\infty}}\left(\left\|\nabla^{2} \boldsymbol{u}\right\|_{L^{2}}+\|\nabla(\rho, \boldsymbol{u}, \boldsymbol{B})\|_{L^{2}}\right)\|\nabla \rho\|_{L^{2}} \\
& +C\|(\rho, \boldsymbol{u})\|_{L^{\infty}}\|\nabla(\rho, \boldsymbol{u})\|_{L^{2}}\|\nabla \boldsymbol{u}\|_{L^{2}} \\
\leq & C\|(\rho, \boldsymbol{u}, \boldsymbol{B})\|_{2}\left(\|\nabla \boldsymbol{u}\|_{1}^{2}+\|\nabla \boldsymbol{B}\|_{L^{2}}^{2}+\|\nabla \rho\|_{L^{2}}^{2}\right) \\
\leq & C \delta\left(\|\nabla \boldsymbol{u}\|_{1}^{2}+\|\nabla \boldsymbol{B}\|_{L^{2}}^{2}+\|\nabla \rho\|_{L^{2}}^{2}\right)
\end{aligned}
$$

which inserted into (3.6) yield

$$
\frac{\beta}{2}\|\nabla \rho\|_{L^{2}}^{2}+\frac{d}{d t}\langle\boldsymbol{u}, \nabla \rho\rangle \leq C\left(\left\|\nabla^{2} \boldsymbol{u}\right\|_{L^{2}}^{2}+\|\nabla \boldsymbol{u}\|_{L^{2}}^{2}+\delta\|\nabla \boldsymbol{B}\|_{L^{2}}^{2}\right) .
$$

Now, multiplying (3.5) by a suitably large constant $K_{1}>0$ and adding it to (3.7), we obtain the desired estimate indicated in (3.2) since $\delta>0$ is small enough.

In the following, we give the proof of (3.3). Applying $D^{k}$ with $1 \leq|k| \leq 3$ to $(1.4)_{1}$, $(1.4)_{2}$, and $(1.4)_{3}$, multiplying the resulting equations by $D^{k} \rho, D^{k} \boldsymbol{u}$, and $D^{k} \boldsymbol{B}$ in $L^{2}$, respectively, and then adding them together, we obtain

$$
\begin{aligned}
& \frac{1}{2} \frac{d}{d t}\|\nabla(\rho, \boldsymbol{u}, \boldsymbol{B})(t)\|_{2}^{2}+\tilde{\lambda}\left\|\nabla^{2} \boldsymbol{u}\right\|_{2}^{2}+\tilde{\mu}\|\nabla \operatorname{div} \boldsymbol{u}\|_{2}^{2}+\nu\left\|\nabla^{2} \boldsymbol{B}\right\|_{2}^{2} \\
= & \left\langle D^{k} f, D^{k} \rho\right\rangle+\left\langle D^{k} \boldsymbol{g}, D^{k} \boldsymbol{u}\right\rangle+\left\langle D^{k} \boldsymbol{h}, D^{k} \boldsymbol{B}\right\rangle .
\end{aligned}
$$

Here and in the following proof, a repeated index denotes summation over the index. 
We now have the task of estimating each term on the right-hand side of (3.8). First, recalling the definition of $f$, we obtain

$$
\begin{aligned}
\left\langle D^{k} f, D^{k} \rho\right\rangle= & -\alpha\left\langle D^{k}(\rho \operatorname{div} \boldsymbol{u}), D^{k} \rho\right\rangle-\alpha\left\langle(\boldsymbol{u} \cdot \nabla) D^{k} \rho, D^{k} \rho\right\rangle \\
& -\alpha\left\langle D^{k}(\boldsymbol{u} \cdot \nabla) \rho-(\boldsymbol{u} \cdot \nabla) D^{k} \rho, D^{k} \rho\right\rangle .
\end{aligned}
$$

Using the product estimate and Sobolev inequalities, we deduce from (3.1) that

$$
\begin{aligned}
\left|\left\langle D^{k}(\rho \operatorname{div} \boldsymbol{u}), D^{k} \rho\right\rangle\right| & \leq C\left(\|\rho\|_{L^{\infty}}\|\operatorname{div} \boldsymbol{u}\|_{3}+\|\rho\|_{3}\|\operatorname{div} \boldsymbol{u}\|_{L^{\infty}}\right)\|\nabla \rho\|_{2} \\
& \leq C\|\rho\|_{3}\|\nabla \boldsymbol{u}\|_{3}\|\nabla \rho\|_{2} \leq C \delta\left(\|\nabla \rho\|_{2}^{2}+\|\nabla \boldsymbol{u}\|_{3}^{2}\right) .
\end{aligned}
$$

After integrating by parts, we infer from Sobolev inequalities and (3.1) that

$$
\left|\left\langle(\boldsymbol{u} \cdot \nabla) D^{k} \rho, D^{k} \rho\right\rangle\right| \leq \frac{1}{2}\|\operatorname{div} \boldsymbol{u}\|_{L^{\infty}}\|\nabla \rho\|_{2}^{2} \leq C \delta\|\nabla \rho\|_{2}^{2} .
$$

By virtue of the commutator estimate and Sobolev inequalities, we obtain

$$
\begin{aligned}
\left|\left\langle D^{k}(\boldsymbol{u} \cdot \nabla) \rho-(\boldsymbol{u} \cdot \nabla) D^{k} \rho, D^{k} \rho\right\rangle\right| & \leq C\left(\|\boldsymbol{u}\|_{3}\|\nabla \rho\|_{L^{\infty}}+\|\nabla \boldsymbol{u}\|_{L^{\infty}}\|\nabla \rho\|_{2}\right)\|\nabla \rho\|_{2} \\
& \leq C\|\boldsymbol{u}\|_{3}\|\nabla \rho\|_{2}^{2} \leq C \delta\|\nabla \rho\|_{2}^{2} .
\end{aligned}
$$

Therefore,

$$
\left|\left\langle D^{k} f, D^{k} \rho\right\rangle\right| \leq C \delta\left(\|\nabla \rho\|_{2}^{2}+\|\nabla \boldsymbol{u}\|_{3}^{2}\right), \quad 1 \leq|k| \leq 3 .
$$

Similarly, we also have that for $1 \leq|k| \leq 3$,

$$
\left|\left\langle D^{k} \boldsymbol{h}, D^{k} \boldsymbol{B}\right\rangle\right| \leq C\|(\boldsymbol{u}, \boldsymbol{B})\|_{3}\|\nabla \boldsymbol{u}\|_{3}\|\nabla \boldsymbol{B}\|_{3} \leq C \delta\left(\|\nabla \boldsymbol{u}\|_{3}^{2}+\|\nabla \boldsymbol{B}\|_{3}^{2}\right) .
$$

Finally, in order to estimate $\left\langle D^{k} \boldsymbol{g}, D^{k} \boldsymbol{u}\right\rangle$, we first notice that

$$
\sum_{|k| \leq 3}\left|D^{k}\left(\frac{1}{\rho+\rho_{\infty}}-\frac{1}{\rho_{\infty}}\right)\right| \sim \sum_{|k| \leq 3}\left|D^{k} \rho\right|+|D \rho|^{2}+|D \rho|^{3}+|D \rho|\left|D^{2} \rho\right|,
$$

which together with Sobolev inequalities and (3.1) leads to

$$
\begin{aligned}
\left\|\left(\frac{1}{\rho+\rho_{\infty}}-\frac{1}{\rho_{\infty}}\right)\right\|_{3} & \leq C\left\{\|\rho\|_{3}+\left(\|\nabla \rho\|_{L^{\infty}}+\|\nabla \rho\|_{L^{\infty}}^{2}\right)\|\nabla \rho\|_{1}\right\} \\
& \leq C\left(1+\|\rho\|_{3}^{2}\right)\|\rho\|_{3} \leq C\|\rho\|_{3} .
\end{aligned}
$$

Thus, similar to the derivation of (3.9), we can utilize Lemma 2.5 to deduce that

$$
\begin{aligned}
& \quad\left|\left\langle D^{k}\left[\left(\frac{\lambda}{\rho+\rho_{\infty}}-\frac{\lambda}{\rho_{\infty}}\right) \Delta \boldsymbol{u}+\left(\frac{\lambda}{\rho+\rho_{\infty}}-\frac{\lambda}{\rho_{\infty}}\right) \nabla \operatorname{div} \boldsymbol{u}\right], D^{k} \boldsymbol{u}\right\rangle\right| \\
& \leq C\left(\|\rho\|_{3}\left\|\nabla^{2} \boldsymbol{u}\right\|_{2}\|\nabla \boldsymbol{u}\|_{2}+\|\rho\|_{L^{\infty}}\|\nabla \boldsymbol{u}\|_{3}^{2}\right) \leq C \delta\|\nabla \boldsymbol{u}\|_{3}^{2} .
\end{aligned}
$$

In the same way, using Lemma 2.5, (3.1), and integrating by parts, we find

$$
\begin{aligned}
& \left|\left\langle D^{k}\left[\left(\frac{p^{\prime}\left(\rho+\rho_{\infty}\right)}{\rho+\rho_{\infty}}-\frac{p^{\prime}\left(\rho_{\infty}\right)}{\rho_{\infty}}\right) \nabla \rho\right], D^{k} \boldsymbol{u}\right\rangle\right| \\
\leq & C\left(\|\rho\|_{3}\|\nabla \rho\|_{L^{\infty}}+\|\nabla \rho\|_{L^{\infty}}\|\nabla \rho\|_{2}\right)\|\nabla \boldsymbol{u}\|_{2}+C\|(\rho, \nabla \rho)\|_{L^{\infty}}\|\nabla \rho\|_{2}\|\nabla \boldsymbol{u}\|_{3} \\
\leq & C\|\rho\|_{3}\|\nabla \rho\|_{2}\|\nabla \boldsymbol{u}\|_{3} \leq C \delta\left(\|\nabla \rho\|_{2}^{2}+\|\nabla \boldsymbol{u}\|_{3}^{2}\right) .
\end{aligned}
$$


By using the product estimate and Sobolev inequalities, it follows from (3.1) that

$$
\left|\left\langle D^{k}(\boldsymbol{u} \cdot \nabla) \boldsymbol{u}, D^{k} \boldsymbol{u}\right\rangle\right| \leq C\left(\|\boldsymbol{u}\|_{L^{\infty}}\|\nabla \boldsymbol{u}\|_{3}+\|\boldsymbol{u}\|_{3}\|\nabla \boldsymbol{u}\|_{L^{\infty}}\right)\|\nabla \boldsymbol{u}\|_{2} \leq C \delta\|\nabla \boldsymbol{u}\|_{3}^{2} .
$$

Due to the product estimate and Sobolev inequalities, we have from (3.1) that

$$
\left\|\left(\rho+\rho_{\infty}\right)^{-1} \boldsymbol{B}\right\|_{3} \leq C\left(1+\|\rho\|_{3}\right)\|\boldsymbol{B}\|_{3} \leq C\|\boldsymbol{B}\|_{3} \leq C \delta,
$$

whence

$$
\left|\left\langle D^{k}\left(\frac{\alpha^{-1}}{\rho+\rho_{\infty}}(\nabla \times \boldsymbol{B}) \times \boldsymbol{B}\right), D^{k} \boldsymbol{u}\right\rangle\right| \leq C \delta\left(\|\nabla \boldsymbol{u}\|_{3}^{2}+\|\nabla \boldsymbol{B}\|_{3}^{2}\right) .
$$

Collecting these estimates together, we arrive at

$$
\left|\left\langle D^{k} \boldsymbol{g}, D^{k} \boldsymbol{u}\right\rangle\right| \leq C \delta\left(\|\nabla \rho\|_{2}^{2}+\|\nabla \boldsymbol{u}\|_{3}^{2}+\|\nabla \boldsymbol{B}\|_{3}^{2}\right), \quad 1 \leq|k| \leq 3 .
$$

With the help of (3.9)-(3.11), we deduce from (3.8) that

$$
\frac{d}{d t}\|\nabla(\rho, \boldsymbol{u}, \boldsymbol{B})(t)\|_{2}^{2}+\left\|\nabla^{2}(\boldsymbol{u}, \boldsymbol{B})(t)\right\|_{2}^{2} \leq C \delta\left(\|\nabla \rho\|_{2}^{2}+\|\nabla(\boldsymbol{u}, \boldsymbol{B})\|_{3}^{2}\right) .
$$

On the other hand, if we apply $D^{l}$ with $1 \leq|l| \leq 2$ to $(1.4)_{2}$, multiply it by $\nabla D^{l} \rho$ in $L^{2}$, and then perform the computations similar to those used in the derivations of (3.7) and (3.12), we can show that

$$
\frac{\beta}{2}\left\|\nabla^{2} \rho\right\|_{1}^{2}+\frac{d}{d t}\left\langle\nabla D^{l} \rho, D^{l} \boldsymbol{u}\right\rangle \leq C\left\|\nabla^{2} \boldsymbol{u}\right\|_{2}^{2}+C \delta\left(\|\nabla \rho\|_{2}^{2}+\|\nabla(\boldsymbol{u}, \boldsymbol{B})\|_{3}^{2}\right) .
$$

Since $\delta>0$ is small enough, multiplying (3.12) by a suitably large constant $K_{2}>0$ and adding it to (3.13) leads to (3.3) at once. This ends the proof of Lemma 3.1.

\subsection{Proof of global existence.}

Proof. It follows from (3.5), (3.7), (3.12), and (3.13) that for enough small $\delta>0$, it holds that

$$
\begin{aligned}
K_{3} \frac{d}{d t}\|(\rho, \boldsymbol{u}, \boldsymbol{B})(t)\|_{3}^{2} & +C_{1} \frac{d}{d t} \sum_{|l| \leq 2}\left\langle\nabla D^{l} \rho, D^{l} \boldsymbol{u}\right\rangle \\
& +C_{2}\left(\|\nabla \rho(t)\|_{2}^{2}+\|\nabla(\boldsymbol{u}, \boldsymbol{B})(t)\|_{3}^{2}\right) \leq 0,
\end{aligned}
$$

for a suitably large constant $K_{3}>0$. Since

$$
\sum_{|l| \leq 2}\left|\left\langle\nabla D^{l} \rho, D^{l} \boldsymbol{u}\right\rangle\right| \leq C\left(\|\rho(t)\|_{3}^{2}+\|\boldsymbol{u}(t)\|_{3}^{2}\right),
$$

choosing $K_{3}>0$ large enough we obtain that

$$
\|(\rho, \boldsymbol{u}, \boldsymbol{B})(t)\|_{3}^{2}+\int_{0}^{t}\left(\|\nabla \rho(\tau)\|_{2}^{2}+\|\nabla(\boldsymbol{u}, \boldsymbol{B})(\tau)\|_{3}^{2}\right) \mathrm{d} \tau \leq K\left\|\left(\rho_{0}, \boldsymbol{u}_{0}, \boldsymbol{B}_{0}\right)\right\|_{3}^{2}
$$

for some positive constant $K>0$. So, if the initial data satisfies $\left\|\left(\rho_{0}, \boldsymbol{u}_{0}, \boldsymbol{B}_{0}\right)\right\|_{3} \leq \varepsilon$ with $\varepsilon>0$ sufficiently small, we have

$$
\|(\rho, \boldsymbol{u}, \boldsymbol{B})(t)\|_{3}^{2} \leq K\left\|\left(\rho_{0}, \boldsymbol{u}_{0}, \boldsymbol{B}_{0}\right)\right\|_{3}^{2} \leq \delta, \quad 0 \leq t \leq T,
$$


which in particular implies that the a priori assumption (3.1) is reasonable. So, the existence of a global-in-time solution $(\rho, \boldsymbol{u}, \boldsymbol{B}) \in H^{3}$ to the initial value problem (1.4) and (1.5) with small initial data follows immediately from the local existence result (see [35] for the study of local existence on more general conversation laws) and the standard continuity argument (see, for example, $[36,37])$. This in turn yields the global existence theorem for the initial value problem (1.1) and (1.2), and completes the proof of Proposition 1.1. Note that, based on the standard energy method, the uniqueness of the solution can be easily proved.

\subsection{Proof of decay estimates.}

Proof. In this subsection, we turn to the proof of the decay estimates. For this purpose, we first infer from Proposition 1.1, (1.6), and (3.3) that the solution $(\rho, \boldsymbol{u}, \boldsymbol{B})$ of the problem (1.4), (1.5) satisfies

$$
\|(\rho, \boldsymbol{u}, \boldsymbol{B})(t)\|_{3} \leq C\left\|\left(\rho_{0}, \boldsymbol{u}_{0}, \boldsymbol{B}_{0}\right)\right\|_{3} \leq C \varepsilon
$$

and

$$
\begin{aligned}
& \frac{d}{d t}\left(K_{2}\|\nabla(\rho, \boldsymbol{u}, \boldsymbol{B})(t)\|_{2}^{2}+\sum_{1 \leq|l| \leq 2}\left\langle\nabla D^{l} \rho, D^{l} \boldsymbol{u}\right\rangle\right) \\
&+C\left(\left\|\nabla^{2} \rho(t)\right\|_{1}^{2}+\left\|\nabla^{2}(\boldsymbol{u}, \boldsymbol{B})(t)\right\|_{2}^{2}\right) \leq C \varepsilon\|\nabla(\rho, \boldsymbol{u}, \boldsymbol{B})(t)\|_{L^{2}}^{2}
\end{aligned}
$$

for some constant $K_{2}>0$ which can be chosen to be sufficiently large. Moreover, using Lemmas 2.2 and 2.4, we find for any given $1 \leq p<6 / 5$ that

$$
\begin{aligned}
\left\|\nabla^{k}(\rho, \boldsymbol{u}, \boldsymbol{B})(t)\right\|_{L^{2}} \leq & C(1+t)^{-\sigma(p, 2 ; k)}\left\|\left(\rho_{0}, \boldsymbol{u}_{0}, \boldsymbol{B}_{0}\right)\right\|_{L^{p} \cap H^{k}} \\
& +C \int_{0}^{t}(1+t-\tau)^{-\sigma(p, 2 ; k)}\|(f, \boldsymbol{g}, \boldsymbol{h})(\tau)\|_{L^{p} \cap H^{k}} d \tau
\end{aligned}
$$

Thanks to the interpolation inequality, Hölder inequality and Sobolev inequalities, we deduce from (3.14) that for any given $1 \leq p<6 / 5$,

$$
\begin{aligned}
\|f\|_{L^{p}} & \leq C\|f\|_{L^{1}}^{\theta}\|f\|_{6 / 5}^{1-\theta} \\
& \leq C\left(\|\rho \operatorname{div} \boldsymbol{u}\|_{L^{1}}+\|(\boldsymbol{u} \cdot \nabla) \rho\|_{L^{1}}\right)^{\theta}\left(\|\rho \operatorname{div} \boldsymbol{u}\|_{L^{6 / 5}}+\|(\boldsymbol{u} \cdot \nabla) \rho\|_{L^{6 / 5}}\right)^{1-\theta} \\
& \leq C\left(\|\rho\|_{L^{2}}\|\nabla \boldsymbol{u}\|_{L^{2}}+\|\boldsymbol{u}\|_{L^{2}}\|\nabla \rho\|_{L^{2}}\right)^{\theta}\left(\|\rho\|_{L^{3}}\|\nabla \boldsymbol{u}\|_{L^{2}}+\|\boldsymbol{u}\|_{L^{3}}\|\nabla \rho\|_{L^{2}}\right)^{1-\theta} \\
& \leq C\left(\|\rho\|_{L^{2}}\|\nabla \boldsymbol{u}\|_{L^{2}}+\|\boldsymbol{u}\|_{L^{2}}\|\nabla \rho\|_{L^{2}}\right)^{\theta}\left(\|\rho\|_{1}\|\nabla \boldsymbol{u}\|_{L^{2}}+\|\boldsymbol{u}\|_{1}\|\nabla \rho\|_{L^{2}}\right)^{1-\theta} \\
& \leq C\|(\rho, \boldsymbol{u})\|_{1}\|\nabla(\rho, \boldsymbol{u})\|_{L^{2}} \leq C \varepsilon\|\nabla(\rho, \boldsymbol{u})\|_{L^{2}}, \quad \theta=(6-5 p) / p .
\end{aligned}
$$

Similarly,

$$
\begin{aligned}
\|\boldsymbol{g}\|_{L^{p}} \leq & C\|\boldsymbol{g}\|_{L^{1}}^{\theta}\|\boldsymbol{g}\|_{6 / 5}^{1-\theta} \\
\leq & C\left(\|\rho\|_{L^{2}}\left\|\nabla^{2} \boldsymbol{u}\right\|_{L^{2}}+\|\boldsymbol{u}\|_{L^{2}}\|\nabla \boldsymbol{u}\|_{L^{2}}+\|\rho\|_{L^{2}}\|\nabla \rho\|_{L^{2}}+\|\boldsymbol{B}\|_{L^{2}}\|\nabla \boldsymbol{B}\|_{L^{2}}\right)^{\theta} \\
& \times\left(\|\rho\|_{L^{3}}\left\|\nabla^{2} \boldsymbol{u}\right\|_{L^{2}}+\|\boldsymbol{u}\|_{L^{3}}\|\nabla \boldsymbol{u}\|_{L^{2}}+\|\rho\|_{L^{3}}\|\nabla \rho\|_{L^{2}}+\|\boldsymbol{B}\|_{L^{3}}\|\nabla \boldsymbol{B}\|_{L^{2}}\right)^{1-\theta} \\
\leq & C\|(\rho, \boldsymbol{u}, \boldsymbol{B})\|_{1}\|\nabla(\rho, \boldsymbol{u}, \boldsymbol{B})\|_{1} \leq C \varepsilon\|\nabla(\rho, \boldsymbol{u}, \boldsymbol{B})\|_{1}
\end{aligned}
$$

and

$$
\|\boldsymbol{h}\|_{L^{p}} \leq C\|\boldsymbol{h}\|_{L^{1}}^{\theta}\|\boldsymbol{h}\|_{6 / 5}^{1-\theta} \leq C\|(\boldsymbol{u}, \boldsymbol{B})\|_{1}\|\nabla(\boldsymbol{u}, \boldsymbol{B})\|_{1} \leq C \varepsilon\|\nabla(\boldsymbol{u}, \boldsymbol{B})\|_{1} .
$$


Consequently,

$$
\|(f, \boldsymbol{g}, \boldsymbol{h})(t)\|_{L^{p}} \leq C \varepsilon\|\nabla(\rho, \boldsymbol{u}, \boldsymbol{B})(t)\|_{1} .
$$

On the other hand, using Sobolev inequalities, we easily obtain

$$
\begin{aligned}
\|(f, \boldsymbol{g}, \boldsymbol{h})\|_{1} & \leq C\|(\rho, \boldsymbol{u}, \boldsymbol{B})\|_{W^{1, \infty}}\|\nabla(\rho, \boldsymbol{u}, \boldsymbol{B})\|_{2} \\
& \leq C\|(\rho, \boldsymbol{u}, \boldsymbol{B})\|_{3}\|\nabla(\rho, \boldsymbol{u}, \boldsymbol{B})\|_{2} \leq C \varepsilon\|\nabla(\rho, \boldsymbol{u}, \boldsymbol{B})\|_{2} .
\end{aligned}
$$

Thus, putting (3.17) and (3.18) into (3.16) with $k=1$, one obtains

$$
\begin{aligned}
\|\nabla(\rho, \boldsymbol{u}, \boldsymbol{B})(t)\|_{L^{2}} \leq & C M_{0}(1+t)^{-\sigma(p, 2 ; 1)} \\
& +C \varepsilon \int_{0}^{t}(1+t-\tau)^{-\sigma(p, 2 ; 1)}\|\nabla(\rho, \boldsymbol{u}, \boldsymbol{B})(\tau)\|_{2} d \tau,
\end{aligned}
$$

where $\varepsilon>0$ is a small constant, and $M_{0}=\left\|\left(\rho_{0}, \boldsymbol{u}_{0}, \boldsymbol{B}_{0}\right)\right\|_{L^{p} \cap H^{1}}$.

With these preparations, we can now prove Proposition 1.5. To this end, we define

$$
M(t)=\sup _{0 \leq \tau \leq t}(1+\tau)^{2 \sigma(p, 2 ; 1)} H(\tau)
$$

where

$$
H(t):=K_{2}\|\nabla(\rho, \boldsymbol{u}, \boldsymbol{B})(t)\|_{2}^{2}+\sum_{1 \leq|l| \leq 2}\left\langle\nabla D^{l} \rho, D^{l} \boldsymbol{u}\right\rangle
$$

with a suitably large constant $K_{2}>0$.

It follows from (3.15) that $H(t)$ satisfies

$$
\frac{d H(t)}{d t}+C\left\|\nabla^{2}(\rho, \boldsymbol{u}, \boldsymbol{B})(t)\right\|_{1}^{2} \leq C \varepsilon\|\nabla(\rho, \boldsymbol{u}, \boldsymbol{B})(t)\|_{L^{2}}^{2} .
$$

Adding $\|\nabla(\rho, \boldsymbol{u}, \boldsymbol{B})(t)\|_{L^{2}}^{2}$ to both sides of this inequality results in

$$
\frac{d H(t)}{d t}+C_{1}\|\nabla(\rho, \boldsymbol{u}, \boldsymbol{B})(t)\|_{2}^{2} \leq C_{2}\|\nabla(\rho, \boldsymbol{u}, \boldsymbol{B})(t)\|_{L^{2}}^{2} .
$$

Since $\left|\left\langle\nabla D^{l} \rho, D^{l} \boldsymbol{u}\right\rangle\right| \leq C\|\nabla(\rho, \boldsymbol{u})\|_{2}^{2}$ for $1 \leq|l| \leq 2$, taking $K_{2}>0$ sufficiently large we find that $H(t)$ is equivalent to $\|\nabla(\rho, \boldsymbol{u}, \boldsymbol{B})(t)\|_{2}^{2}$, that is, there exists a constant $C>1$ such that

$$
C^{-1}\|\nabla(\rho, \boldsymbol{u}, \boldsymbol{B})(t)\|_{2}^{2} \leq H(t) \leq C\|\nabla(\rho, \boldsymbol{u}, \boldsymbol{B})(t)\|_{2}^{2},
$$

from which we see that

$$
\frac{d H(t)}{d t}+C_{1} H(t) \leq C_{2}\|\nabla(\rho, \boldsymbol{u}, \boldsymbol{B})(t)\|_{L^{2}}^{2} .
$$

To deal with the term on the right-hand side of (3.22), we utilize (3.20), (3.21), and Lemma 2.6 to get from (3.19) that

$$
\begin{aligned}
\|\nabla(\rho, \boldsymbol{u}, \boldsymbol{B})(t)\|_{L^{2}} \leq & C M_{0}(1+t)^{-\sigma(p, 2 ; 1)} \\
& +C \varepsilon \sqrt{M(t)} \int_{0}^{t}(1+t-\tau)^{-\sigma(p, 2 ; 1)}(1+\tau)^{-\sigma(p, 2 ; 1)} d \tau \\
\leq & C(1+t)^{-\sigma(p, 2 ; 1)}\left(M_{0}+\varepsilon \sqrt{M(t)}\right),
\end{aligned}
$$


since $M(t)$ is non-decreasing in $t$ and $\sigma(p, 2 ; 1)>1$ when $1 \leq p<6 / 5$.

Putting (3.23) into (3.22) and keeping in mind that $M(t)$ is non-decreasing, we have from Lemma 2.6 that

$$
\begin{aligned}
H(t) & \leq H(0) e^{-C_{1} t}+C\left(M_{0}^{2}+\varepsilon^{2} M(t)\right) \int_{0}^{t}(1+\tau)^{-2 \sigma(p, 2 ; 1)} e^{-C_{1}(t-\tau)} d \tau \\
& \leq C(1+t)^{-2 \sigma(p, 2 ; 1)}\left(H(0)+M_{0}^{2}+\varepsilon^{2} M(t)\right) .
\end{aligned}
$$

So, if $\varepsilon>0$ is taken to be small enough, then it follows from (3.24) and (3.20) that

$$
M(t) \leq C\left(H(0)+M_{0}^{2}\right),
$$

which combined with (3.21) and (3.24) implies that

$$
\|\nabla(\rho, \boldsymbol{u}, \boldsymbol{B})(t)\|_{2} \leq C \sqrt{H(t)} \leq C(1+t)^{-\sigma(p, 2 ; 1)}, \quad \forall t \geq 0 .
$$

This completes the proof of (1.8).

To obtain the decay estimates of $\|(\rho, \boldsymbol{u}, \boldsymbol{B})(t)\|_{L^{q}}(2 \leq q \leq 6)$, we first utilize (3.17), (3.18), (3.25), and Lemma 2.6 to infer from (3.16) that

$$
\begin{aligned}
\|(\rho, \boldsymbol{u}, \boldsymbol{B})(t)\|_{L^{2}} & \leq C M_{0}(1+t)^{-\sigma(p, 2 ; 0)}+C \varepsilon \int_{0}^{t}(1+t-\tau)^{-\sigma(p, 2 ; 0)}\|\nabla(\rho, \boldsymbol{u}, \boldsymbol{B})(\tau)\|_{2} d \tau \\
& \leq C M_{0}(1+t)^{-\sigma(p, 2 ; 0)}+C \varepsilon \int_{0}^{t}(1+t-\tau)^{-\sigma(p, 2 ; 0)}(1+\tau)^{-\sigma(p, 2 ; 1)} d \tau \\
& \leq C(1+t)^{-\sigma(p, 2 ; 0)}
\end{aligned}
$$

On the other hand, using Sobolev's inequality and (3.25), we find

$$
\|(\rho, \boldsymbol{u}, \boldsymbol{B})(t)\|_{L^{6}} \leq\|\nabla(\rho, \boldsymbol{u}, \boldsymbol{B})(t)\|_{L^{2}} \leq C(1+t)^{-\sigma(p, 2 ; 1)} .
$$

By virtue of (3.26) and (3.27), we have from the interpolation inequality that

$$
\begin{aligned}
\|(\rho, \boldsymbol{u}, \boldsymbol{B})(t)\|_{L^{q}} & \leq\|(\rho, \boldsymbol{u}, \boldsymbol{B})(t)\|_{L^{2}}^{(6-q) /(2 q)}\|(\rho, \boldsymbol{u}, \boldsymbol{B})(t)\|_{L^{6}}^{3(q-2) /(2 q)} \\
& \leq C(1+t)^{-\sigma(p, q ; 0)}, \quad 2 \leq q \leq 6
\end{aligned}
$$

This proves the desired decay estimates indicated in (1.10).

Finally, using (3.18) and (3.25), it is easy to see from (1.4) that

$$
\begin{aligned}
\left\|\partial_{t}(\rho, \boldsymbol{u}, \boldsymbol{B})(t)\right\|_{L^{2}} & \leq C\|\nabla(\rho, \boldsymbol{u}, \boldsymbol{B})(t)\|_{1}+\|(f, \boldsymbol{g}, \boldsymbol{h})\|_{L^{2}} \\
& \leq C\|\nabla(\rho, \boldsymbol{u}, \boldsymbol{B})(t)\|_{2} \leq C(1+t)^{-\sigma(p, 2 ; 1)}
\end{aligned}
$$

and

$$
\begin{aligned}
\left\|\partial_{t} \nabla(\rho, \boldsymbol{u}, \boldsymbol{B})(t)\right\|_{L^{2}} & \leq C\|\nabla(\rho, \boldsymbol{u}, \boldsymbol{B})(t)\|_{2}+\|\nabla(f, \boldsymbol{g}, \boldsymbol{h})\|_{L^{2}} \\
& \leq C\|\nabla(\rho, \boldsymbol{u}, \boldsymbol{B})(t)\|_{2} \leq C(1+t)^{-\sigma(p, 2 ; 1)} .
\end{aligned}
$$

Consequently,

$$
\left\|\partial_{t}(\rho, \boldsymbol{u}, \boldsymbol{B})(t)\right\|_{1} \leq C(1+t)^{-\sigma(p, 2 ; 1)},
$$

which proves the decay estimate in (1.9). Therefore, the proof of Proposition 1.5 is complete, and so is Theorem 1.2.

Acknowledgement. The authors would like to thank the referees for their comments. 


\section{REFERENCES}

[1] R.A. Adams, Sobolev Space, New York, Academic Press, 1975.

[2] J. Bergh and J. Löfstöm, Interpolation Spaces, New York, Spinger-Verlag, 1976.

[3] H. Cabannes, Theoretical Magnetofluiddynamics, New York, Academic Press, 1970.

[4] G.Q. Chen and D. Wang, Global solution of nonlinear magnetohydrodynamics with large initial data, J. Differ. Equs., 182, 344-376, 2002.

[5] G.Q. Chen and D. Wang, Existence and continuous dependence of large solutions for the magnetohydrodynamic equations, Z. Angew. Math. Phys., 54, 608-632, 2003.

[6] K. Deckelnick, Decay estimates for the compressible Navier-Stokes equations in unbounded domains, Math. Z., 209, 115-130, 1992.

[7] K. Deckelnick, $L^{2}$-decay for the compressible Navier-Stokes equations in unbounded domains, Commun. Part. Differ. Equs., 18, 1445-1476, 1993.

8] R.J. Duan, H.X. Liu, S. Ukai and T. Yang, Optimal $L^{p}-L^{q}$ convergence rates for the compressible Navier-Stokes equations with potential force, J. Differ. Equs., 238, 220-233, 2007.

9] R.J. Duan, S. Ukai, T. Yang and H.J. Zhao, Optimal convergence rates for the compressible Navier-Stokes equations with potential forces, Math. Models Meth. Appl. Sci., 17, 737-758, 2007.

[10] B. Ducomet and E. Feireisl, The equations of magnetohydrodynamics: on the interaction between matter and radiation in the evolution of gaseous stars, Commun. Math. Phys., 266, 595-629, 2006.

[11] J. Fan, S. Jiang and G. Nakamura, Vanishing shear viscosity limit in the magnetohydrodynamic equations, Commun. Math. Phys., 270, 691-708, 2007.

[12] J. Fan and W. Yu, Global variational solutions to the compressible magnetohydrodynamic equations, Nonlinear Anal., 69, 3637-3660, 2008.

[13] J. Fan and W. Yu, Strong solution to the compressible magnetohydrodynamic equations with vacuum, Nonlinear Anal. Real World Appl., 10, 392-409, 2009.

[14] E. Feireisl, Dynamics of Viscous Compressible Fluids, Oxford, Oxford University Press, 2004.

[15] D.A. Gurnett and A. Bhattacharjee, Introduction to Plasma Physics, Cambridge, Cambridge Univ. Press, 2005.

[16] D. Hoff and K. Zumbrun, Multi-dimensional diffusion waves for the Navier-Stokes equations of compressible flows, Indiana Univ. Math. J., 44, 603-676, 1995.

[17] D. Hoff and K. Zumbrun, Pointwise decay estimates for multidimensional Navier-Stokes diffusion waves, Z. Angew. Math. Phys., 48, 597-614, 1997.

[18] X. Hu and D. Wang, Global solutions to the three-dimensional full compressible magnetohydrodynamic flows, Commun. Math. Phys., 283, 255-284, 2008.

[19] X. Hu and D. Wang, Compactness of weak solutions to the three-dimensional compressible magnetohydrodynamic equations, J. Differ. Equs., 245, 2176-2198, 2008.

[20] X. Hu and D. Wang, Global existence and large-time behavior of solutions to the threedimensional equations of compressible magnetohydrodynamic flows, Arch. Rational Mech. Anal., to appear, 2009.

[21] Y. Kagei and T. Kobayashi, On large time behavior of solutions to the compressible NavierStokes equations in the half space in $\mathbb{R}^{3}$, Arch. Rational Mech. Anal., 165, 89-159, 2002.

[22] Y. Kagei and T. Kobayashi, Asymptotic behavior of solutions of the compressible Navier-Stokes equations on the half space, Arch. Rational Mech. Anal., 177, 231-330, 2005.

[23] S. Kawashima, Smooth global solutions for two-dimensional equations of electromagneto-fluid dynamics, Japan J. Appl. Math., 1, 207-222, 1984.

[24] S. Kawashima and M. Okada, Smooth global solutions for the one-dimensional equations in magnetohydrodynamics, Proc. Japan Acad. Ser. A Math. Sci. 58, 384-387, 1982.

[25] S. Kawashima and Y. Shizuta, Magnetohydrodynamic approximation of the complete equations for an electromagnetic fluid, Tsukuba J. Math., 10, 131-149, 1986.

[26] S. Kawashima and Y. Shizuta, Magnetohydrodynamic approximation of the complete equations for an electromagnetic fluid II, Proc. Japan Acad. Ser. A, 62, 181-184, 1986.

[27] V. Kazhikhov and V.V. Shelukhin, Unique global solution with respect to time of initialboundary-value problems for one-dimensional equations of a viscous gas, J. Appl. Math. Mech., 41, 273-282, 1977.

[28] T. Kobayashi, Some estimates of solutions for the equations of motion of compressible viscous fluid in the three-dimensional exterior domain, J. Differ. Equs., 184, 587-619, 2002.

[29] T. Kobayashi and Y. Shibata, Decay estimates of solutions for the equations of motion of compressible viscous and heat-conductive gases in an exterior domain in $\mathbb{R}^{3}$, Commun. Math. Phys., 200, 621-659, 1999.

[30] A.F. Kulikovskiy and G.A. Lyubimov, Magnetohydrodynamics, Reading MA, Addison-Wasley, 
1965.

[31] L.D. Landau, E.M. Lifshitz and L.P. Pitaevskii, Electrodynamics of Continuous Media, 2nd (ed.), London, Butterworth-Heinemann, 1999.

[32] T.T. Li and Y. Chen, Global Classical Solutions for Nonlinear Evolution Equations, Harlow, Longman Sci. Tech., 1992.

[33] P.L. Lions, Mathematical Topics in Fluid Dynamics, Compressible Models, Oxford, Oxford Science Publication, 2, 1996.

[34] T.P. Liu and Y. Zeng, Compressible Navier-Stokes equations with zero heat conductivity, J. Differ. Equs., 53, 225-291, 1999.

[35] A. Majda, Compressible Fluid Flow and Systems of Conservation Laws in Several Space Variables, New York, Springer, 1984.

[36] A. Matsumura and T. Nishida, The initial value problem for the equations of motion of viscous and heat-conductive gases, J. Math. Kyoto Univ., 20, 67-104, 1980.

[37] A. Matsumura and T. Nishida, Initial-boundary value problems for the equations of motion of compressible viscous and heat-conductive fluids, Commun. Math. Phys., 89, 445-464, 1983.

[38] A. Novotný and I. Straškraba, Introduction to the Mathematical Theory of Compressible Flow, Oxford, Oxford University Press, 2004.

[39] F. Treves, Basic Linear Partial Differential Equations, New York-London, Academic Press, 1975.

[40] S. Ukai, T. Yang and H.J. Zhao, Convergence rate for the compressible Navier-Stokes equations with external force, J. Hyperbolic Differ. Equs., 3, 561-574, 2006.

[41] D. Wang, Large solutions to the initial-boundary value problem for planar magnetohydrodynamics, SIAM J. Appl. Math., 63, 1424-1441, 2003.

[42] L.C. Woods, Priciples of Magnetoplasma Dynamics, Oxford, Oxford University Press, 1987.

[43] J.-W. Zhang, S. Jiang and F. Xie, Global weak solutions of an initial boundary value problem for screw pinches in plasma physics, Math. Models Meth. Appl. Sci., 19, 833-875, 2009. 